\title{
wayOU - Linked Data-Based Social Location Tracking in a Large, Distributed Organisation
}

\author{
Mathieu d'Aquin, Fouad Zablith, and Enrico Motta \\ Knowledge Media Institute, The Open University, Milton Keynes, UK \\ \{m.daquin,f.zablith, e.motta\}@open.ac.uk
}

\begin{abstract}
While the publication of linked open data has gained momentum in large organisations, the way for users of these organisations to engage with these data is still unclear. Here, we demonstrate a mobile application called wayOU (where are you at the Open University) which relies on the data published by The Open University (under data.open.ac.uk) to provide social, location-based services to its students and members of staff. An interesting aspect of this application is that, not only it consumes linked data produced by the University from various repositories, but it also contributes to it by creating new connections between people, places and other types of resources.
\end{abstract}

\section{Introduction}

The Open University 1 is a large UK University dedicated to distance learning. Apart from its main campus, it is distributed over 13 regional centres across the country. As part of the LUCERO project (Linking University Content for Education and Research Online2), the Open University is publishing linked open data, concerning people, publications, courses, places, and open educational material from existing institutional repositories and databases, under data.open.ac.uk 3

While the collection, conversion, exposure and maintenance of linked data in large organisations is slowly becoming easier, it is still an issue to get users of these organisations to engage with the data in a way suitable to them and that could also benefit to re-enforcing the data. Many 'applications' of linked data mostly concern the visualisation or exploration of available data for a particular purpose (see for example [1]), especially in mobile applications (see for example [2] or 3]), or the use of linked data to accomplish a specific task (e.g., recommendation in DBrec [4]). Our goal is to provide features to users that not only make use of linked data, but which usage would contribute in creating new connections in the data, including currently implicit relations between people and places.

We demonstrate wayOU (where are you at the Open University): a mobile application developed for the Google Android platform 4 that allows users of the

\footnotetext{
1 http://www.open.ac.uk

2 http://lucero-project.info

3 http://data.open.ac.uk

4 http://www.android.com

G. Antoniou et al. (Eds.): ESWC 2011, Part II, LNCS 6644, pp. 461465 2011.

(C) Springer-Verlag Berlin Heidelberg 2011
} 
Open University (members of staff and students) to keep track of the places in which they have been on the main campus and the regional centres, and connect this information to other aspects of their interaction with the organisation (their workplace, meetings with colleagues, tutorials, etc.) This application relies on the data available through the SPARQL endpoint of data.open.ac.uk to get information regarding places (buildings, floors) and people (identifier, network), as well as the linked data published by the UK government regarding locations in the UK (especially, data.ordnancesurvey.co.uk 5). More importantly, it generates and keeps track of information generated for each user regarding their current location, usual workplace, history of visits at the Open University and the reasons for these visits. In this way, it can be compared to the foursquare application 6 working in a specific 'corporate' environment and allowing to declare and expose more complex connections between users and places in this environment.

In the next section, we give a general overview of the structure of the wayOU application and in Section 3, we give more details about the way users interact with it. We discuss in Section 4 the future work and challenges we identified from our experience in building a social, mobile application based on linked data.

\section{The wayOU Android Application}

An overview of the architecture of the wayOU application is presented in Figure 1. The application can run on any Android enabled device (with at least Android OS 1.6 installed). This part of the application is mainly in charge of the interaction between the application and the user. It does not store any information locally (apart from basic settings such as the user's identifier). The information used and produced by the application is manipulated through an ad-hoc SPARQL client, accessing 2 SPARQL endpoints:

data.open.ac.uk provides an open SPARQL endpoint with information regarding the places in the Open University's campus and regional centres, especially regarding buildings and floors within buildings. It also contains information regarding members of the Open University (especially publications and projects), as well as about other resources such as courses, audio/video podcasts, open educational resources, etc. The SPARQL endpoint from data.ordnancesurvey.co.uk is used to obtain information regarding the location of buildings, based on their postcodes 7

A personal information store is used to store the information generated by the application for each user, and to query it. It deploys a service to update the store with RDF regarding the current location and activities of users and a SPARQL endpoint which identifies the appropriate graph to query depending on the identity of the user.

In addition, an option is available to export information from the private space of the personal information store, to the public one of data.open.ac.uk.

\footnotetext{
${ }^{5}$ http://data.ordnancesurvey.co.uk

6 http://foursquare.com

7 Postcode units in the UK identify small areas, often corresponding to a single street.
} 


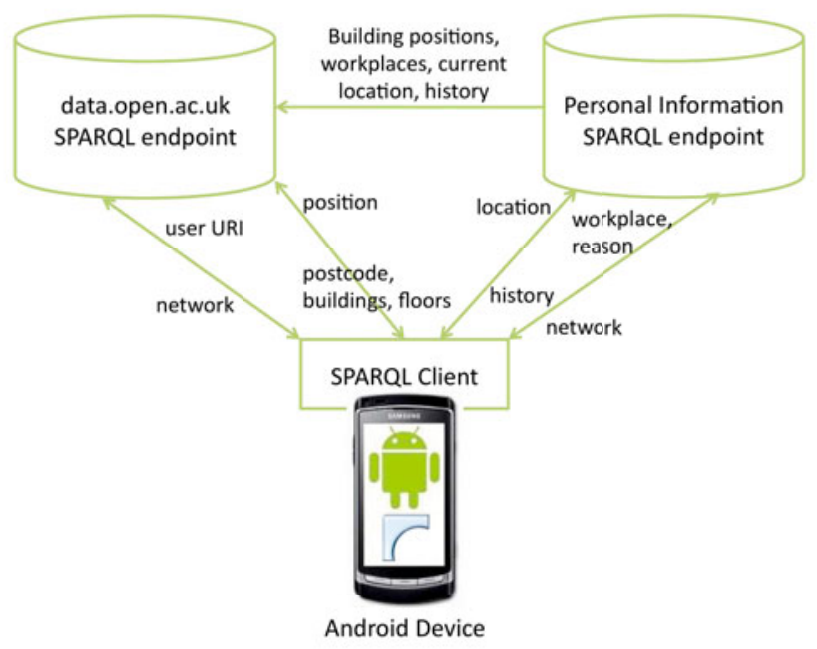

Fig. 1. Overview of the architecture of the wayOU Android application

\section{Interface and Demonstration}

In this section, we quickly go through the mobile interface of the wayOU application, as a way to explain its features and behaviour.

wayOU appears as a standard application on an Android-enabled mobile device. It does not require any setting other than the identifier of the user to work (the OUCU, Open University Computer Username, which is assigned to every member of staff and student of the University. See below).

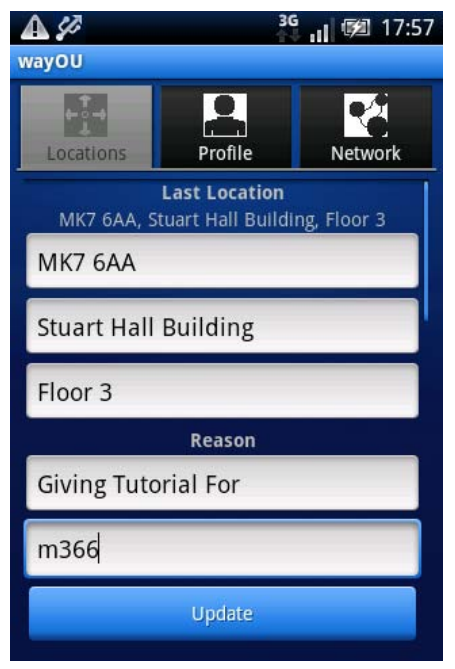

The Locations tab is the main view of the application. It allows to enter the postcode, building name and floor level where the user is currently located. The postcode is automatically set to the closest postcode in which the Open University has buildings. Each field provides suggestions for auto-completion based on relevant data fetched from data.open.ac.uk: the building field suggests buildings in the chosen postcode and the floor field depends on the building field. In this view, it is also possible to provide a reason for attending a specific place. Predefined reasons are given with auto-completion, and include "Meeting with" somebody, "Giving a Tutorial" for a course and "Attending a tutorial" for a course. The second field provides values for autocompletion from the list of courses at The Open University or the list of people in the user's network, depending on the chosen reason. 

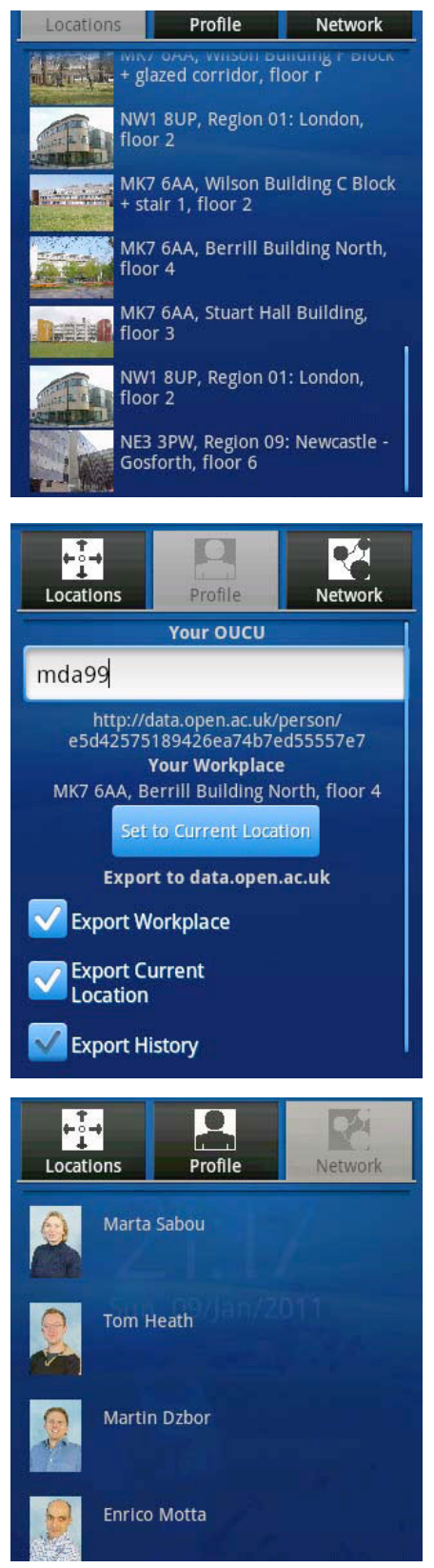

The history of all the places attended by the user is also displayed as a list in the Locations tab. In this list, the user can quickly check whether he or she has already been in a given place, using the provided summary (postcode, building name, floor level) and the picture automatically attached to each item in the list. Each item links to a page describing the relationship between the user and this particular location, including the times he or she has attended it, whether or not it is his/her workplace, and reasons for attending otherwise.

The profile tab allows the user to connect the location information generated from the application to his/her identity at The Open University. The OUCU corresponds to the login of the user on the OU network, and is used to generate the URI identifying this particular user in the data.open.ac.uk linked data space. The user is also offered the possibility to declare his/her current location as his/her workplace, as well as to export the personal information generated by the application into the data.open.ac.uk space. This corresponds to exposing this information as public, so that it can be reused, for example, by the applications generating profile pages for members of staff of the University.

The notion of network is included in the application as a similar mechanism to the ones of 'friends' or 'contacts' in other social applications. Here, an initial list of members of the social network is populated from the list of collaborators of the user, derived from the co-authors of his/her papers, and people at the Open University working, or having worked, on the same projects. In addition, new members of the network can be derived based on the data generated by the application, because of users attending the same meetings or tutorials.

\section{Conclusion, Future Work and Challenges}

The wayOU application is currently being tested with selected users of the Open University. It will be made progressively available to more users before being fully opened, by the time of the conference. An interesting aspect of the wayOU 
application is that it both relies on and contributes to a base of linked data in a given organisation, and from which more information can be derived, possibly leading to new applications (e.g., analysing the movement of people within the organisation depending on their social network).

There are obvious challenges in building linked data-based mobile applications: while the mobile device is not required to realise complex computations, the overhead created by communicating with SPARQL endpoints represents a bottleneck. As the history of locations and the network of users grow, more and more time is needed to obtain and transfer the information. This and other challenges need be investigated in the future:

Dealing with complex privacy options. At the moment, the privacy options permitted by the application are rather simple: everything is private unless explicitly exported to data.open.ac.uk. More complex settings would be desirable, up to the possibility to define arbitrary complex access policies (for example: "give access to my current location on the campus, but not the regional centres, to people I have written papers with in the last 3 years").

Integrating with other social platforms. An obvious next step for this application is the integration with other social platforms, including for example exporting the current location to Twitter 8 or extending the user's network based on his/her Facebook9 account. The Open University has developed several social network applications, including the Facebook course profile application 10 which could enhance and be enhanced by wayOU. Integrating such additional sources of information requires more effort as they are not based on linked data, and would make even more complex the privacy related issues described above.

A generic, reusable application. wayOU is developed specifically for users at the Open University and relies on linked data resources present at the Open University. However, adapting it for other organisations could be envisaged. Since it is designed to use the specific resources, vocabularies and URI Schemes of data.open.ac.uk, it is still unclear how much effort would be required.

\section{References}

1. Lehmann, J., Knappe, S.: DBpedia Navigator. In: ISWC Billion Triple Challenge (2008)

2. Becker, C., Bizer, C.: DBpedia mobile: A location-enabled linked data browser. In: Proceedings of Linked Data on the Web Workshop (LDOW 2008), Citeseer (2008)

3. van Aart, C., Wielinga, B., van Hage, W.R.: Mobile Cultural Heritage Guide: Location-Aware Semantic Search. In: Cimiano, P., Pinto, H.S. (eds.) EKAW 2010. LNCS, vol. 6317, pp. 257-271. Springer, Heidelberg (2010)

4. Passant, A., Decker, S.: Hey! Ho! Let's Go! Explanatory Music Recommendations with dbrec. In: Aroyo, L., Antoniou, G., Hyvönen, E., ten Teije, A., Stuckenschmidt, H., Cabral, L., Tudorache, T. (eds.) ESWC 2010. LNCS, vol. 6089, pp. 411-415. Springer, Heidelberg (2010)

\footnotetext{
8 http://twitter.com

${ }^{9}$ http://facebook.com

${ }^{10}$ http://www . facebook. com/apps/application.php? api_key=06d85b85540794e2fd02e9ef83206bf 6
} 